\title{
ON THE APPROACH TO MAXWELLIAN DISTRIBUTION
}

\author{
J.A. TJON \\ Institute for Theoretical Physics, University of Utrecht, Utrecht, The Netherlands
}

Received 31 January 1979

The relaxation to equilibrium of a gas governed by the Boltzmann equation is studied for various collision models. For certain initial distributions evidence is presented that it can be characterized by a state in which there is an over-population of high-velocity particles.

In a recent paper [1], to be referred to as $I$, the relaxation towards statistical equilibrium of Maxwell molecules in a homogeneous isotropic system has been studied within the framework of the non-linear Boltzmann equation. Numerical evidence has been presented there that for a certain class of initial states the distribution function $f(v, \tau)$ first tends towards a mode which is a similarity solution and in the subsequent stage the system approaches its final state along this mode. In this note we extend these calculations and also present results for non-Maxwell molecules. Evidence is given here that there are initial states which relax towards equilibrium in a qualitatively different way as discussed previously.

Our starting point is the Boltzmann equation for the case that the scattering between the particles is diffusive, but still satisfying the conservation of total energy in the collision process. Following the notation of $I$, we assume that the collision matrix $A$ for a $d$ dimensional system is given by

$\left.A=\frac{a_{0}}{\left(v^{2}+w^{2}\right.}\right)^{d-1} g\left(v^{2}+w^{2}\right) \delta\left(v^{2}+w^{2}-v^{\prime 2}-w^{\prime 2}\right)$.

Confining ourselves further to the $d=2$ case, the Boltzmann equation can be written in the following form:

$$
\begin{gathered}
\frac{\partial F(x, \tau)}{\partial \tau}+\int_{0}^{\infty} \frac{\mathrm{d} x^{\prime}}{x+x^{\prime}} g\left(x+x^{\prime}\right) \int_{0}^{x+x^{\prime}} \mathrm{d} x^{\prime \prime}\left[F(x, \tau) F\left(x^{\prime}, \tau\right)\right. \\
\left.-F\left(x+x^{\prime}-x^{\prime \prime}, \tau\right) F\left(x^{\prime \prime}, \tau\right)\right]=0
\end{gathered}
$$

where $F(x, \tau)=f(v, \tau)$ with $x=v^{2} / 2$. As in $\mathrm{I}$, in order to study the numerical solutions to eq. (2), the integrations over $x$ are carried out with the trapezoidal rule and the time derivative is replaced by a time difference with step size $\Delta \tau$. The resulting equations are

$$
\begin{gathered}
F_{i}(\tau+\Delta \tau)=F_{i}(\tau)+\Delta \Delta \tau \sum_{k=i}^{N-1} \epsilon_{k-i} k^{-1} g_{k} \\
\times \sum_{j=0}^{k} \epsilon_{j} \epsilon_{k-j}\left[F_{j}(\tau) F_{k-j}(\tau)-F_{i}(\tau) F_{k}(\tau)\right],
\end{gathered}
$$

with $x=j \Delta, F_{j}(\tau)=F(j \Delta, \tau)$ and $g_{k}=g(k \Delta)$. The solutions of eq. (3) have the nice properties that the number $N(\tau)=\Delta \Sigma_{n} \epsilon_{n} F_{n}(\tau)$ and the average energy $E(\tau)=\Delta^{2} \Sigma_{n} n F_{n}(\tau)$ are exactly conserved. Furthermore, the $\mathrm{H}$-theorem is satisfied.

The case of Maxwell molecules, corresponding to $g_{n}=1$, has been studied in detail in I. In particular, it was established from $\chi^{2}$ fits, that the approach to equilibrium of an initial state, for which $N(\tau)=E(\tau)$ $=1$ and $F_{n}(0)=0$ except at the points $\left(n_{1}, n_{2}\right)=$ $\left(0, n_{2}\right)$ with $n_{2} \Delta<2$, is governed after a short time by the similarity solution found by Bobylev [2] and Krook and Wu $[3,4]$. More recently, the case of $g(x)$ $=x$ has been discussed by Ernst and Hendriks [5]. The calculations of I have been extended to the situation where the non-zero energy particles $n_{2}$ have higher energies. It was found that another type of relaxation sets in. As a typical example, in fig. 1 is shown the time dependence of the normalized distribution function $R_{n}(\tau)=F_{n}(\tau) / F_{n}(\infty)$ for the case that 


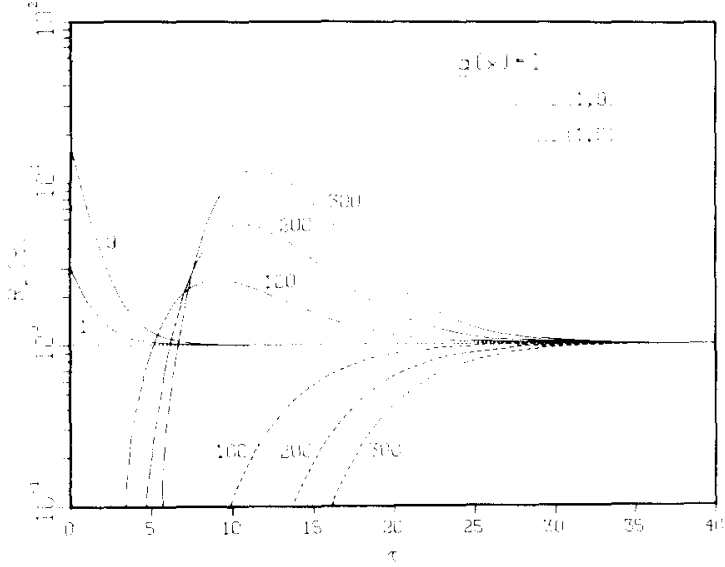

Fig. 1. The normalized distribution $R_{n}$ as a function of time $\tau$ in the case of Maxwell molecules for various $n$. The two initial distributions are given by $F_{n}=0$ except for $n=1,5$ respectively $n=1,9$.

$\left(n_{1}, n_{2}\right)=(1,9)$. To minimize boundary effects we have chosen a comparatively large $N=400$, while the parameters $\Delta$ and $\Delta \tau$ have been taken to be $40 / 99$ and 0.05 , respectively. From fig. 1 we see that the particles which were initially present are relaxing to equilibrium within a time $\tau \approx 6$. Moreover, we see that once the higher-energy particles are produced. the rate of production is very fast, while $R_{n}(\tau)$ keeps increasing beyond 1 . After reaching a maximum value, the approach to equilibrium takes place from above the maxwellian value at a slower rate. For comparison is shown the case that the initial distribution is given by $\left(n_{1}, n_{2}\right)=(1,5)$ for which the relaxation is governed by the similarity mode. In fig. 2 is shown the $n$-dependence of $R_{n}(\tau)$ at various times. For $\left(n_{1}, n_{2}\right)=(1,9)$ the over-population of the high-velocity parts in the distribution function is reached for all considered values of $n$ after a time of order 10 .

The occurrence of an excess of high-energy particles is also found in the special solution [3], which is asymptotically valid for the case that the binary collisions are characterized by an elastic cross section proportional to $|\boldsymbol{v}-\boldsymbol{w}|^{-3}$. We have also studied with our equations a similar collision model by taking $g_{n}$ in eq. (3) of the form

$g_{n}=\left[0.1+\left(\Delta^{\prime} n\right)^{m}\right]^{-1}$,

with $\Delta^{\prime}=\Delta / 4$ and $m=1$. In addition, the case with $m=2$ has also been considered. In these models col-

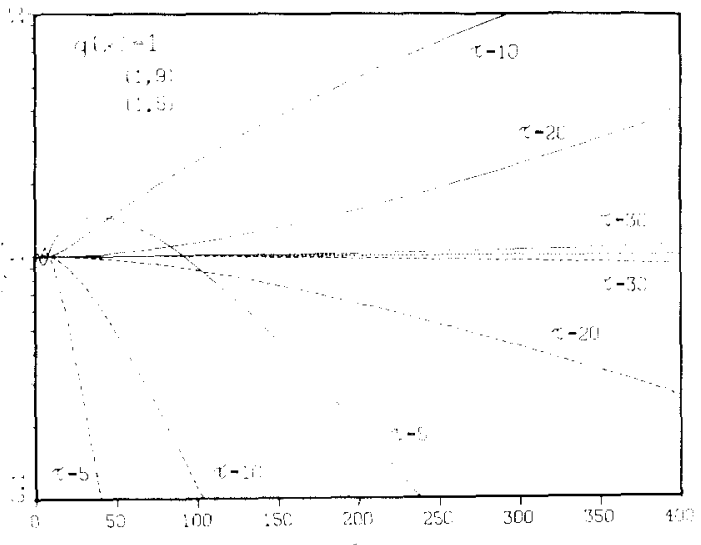

Fig. 2. The same as in fig. 1 , but now as a function of $n$ for various $\tau$.

lisions involving zero-energy particles are favoured more strongly. This class of collision processes are less effective to establish equilibrium. As a result, the relaxation is expected to be slower as compared to the case of Maxwell molecules. This is borne out by the actual calculations. Also, in these cases we have found for sufficiently small $n_{2}$ in the initial distribution that the normalized distribution function $R_{n}(\tau)$ remains below 1 for large $n$. On increasing $n_{2}$ we get an overpopulation of the high-velocity parts. Moreover, the particles with higher velocities take a larger time to reach the equilibrium value. The effect is stronger the larger $m$ is. Some results are shown in

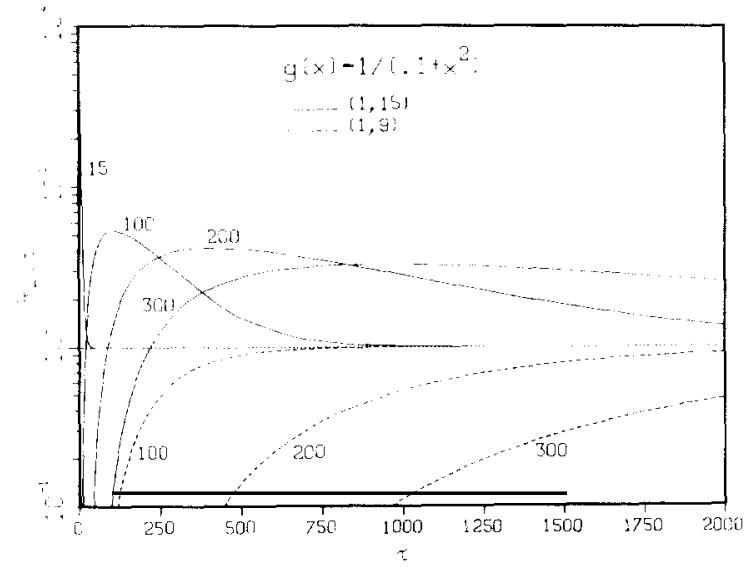

Fig. 3. The normalized distribution $R_{n}$ as a function of time $\tau$ for the collision model $m=2$. The two initial distributions are given by $F_{n}=0$ except for $n=1,9$, respectively $n=1,15$. 


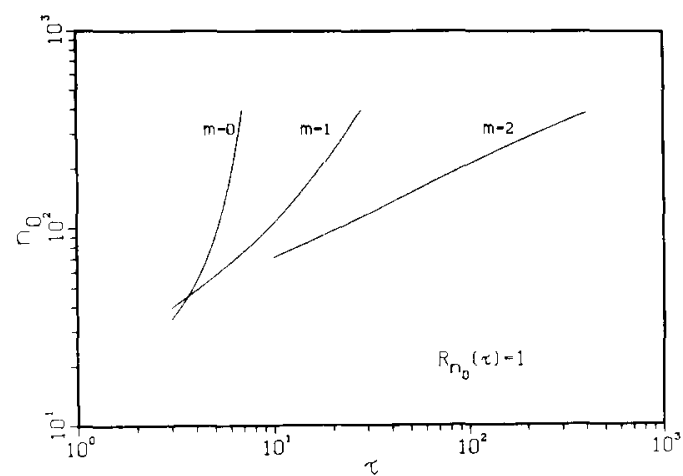

Fig. 4. The dependence of $n_{0}$ for which $R_{n_{0}}(\tau)=1$ as a function of time $\tau$ for the various collision models characterized by $m$.

fig. 3 for the case that $m=2$. From this we see that the time for which $R_{n}(\tau)$ for a given $n$ reaches the maximum value moves to the right for larger $n$, while this maximum decreases slowly as a function of $n$. The magnitude of the over-population of the highvelocity parts increases very strongly if we choose a larger value of $n_{2}$ in the initial state.

As a measure for the spreading of the over-population in the tail of the distribution function we may use the time dependence of the quantity $n_{0}$ defined as the largest value of $n$ for which $R_{n}(\tau)=1$. Some results are plotted in fig. 4 for Maxwell molecules $(m=0)$ and the collision models with $m=1,2$. We see that in all three cases $n_{0}$ keeps increasing as a function of $\tau$. For Maxwell molecules $n_{0}$ diverges exponentially fast for large $\tau$, while it has a fractional power dependence $n_{0}=c \tau^{\alpha}$ with $\alpha=0.46$ in the $m=2$ case. The exponent $\alpha$ turns out, however, not to be universal, but depends on the initial distribution. The above results suggest that the over-population of the high-velocity parts in the distribution also persists at higher velocities. In that case, the conjecture about the similarity mode as considered in I has to be modified as being only valid for a certain class of initial states. Needless to say, numerical studies such as we have undertaken here can, of course, not be considered as definite proof that the discussed behaviour also applies for asymptotic large velocities.

\section{References}

[1] J.A. Tjon and T.T. Wu, Phys. Rev. A, to be published. [2] A.V. Bobylev, Sov. Phys. Dokl. 20 (1976) 820, 822.

[3] M. Krook and T.T. Wu, Phys. Rev. Lett. 36 (1976) 1107.

[4] M. Krook and T.T. Wu, Phys. Fluids 20 (1977) 1589.

[5] M.H. Ernst and E.M. Hendriks, Phys. Lett. 70A (1979) 183. 